\title{
Theoretical Photochemistry of the Photochromic Molecules Based on Density Functional Theory Methods
}

\author{
Ivan A. Mikhailov ${ }^{1}$ and Artëm E. Masunov ${ }^{1,2,3}$ \\ ${ }^{1}$ NanoScience Technology Center \\ ${ }^{2}$ Department of Chemistry \\ ${ }^{3}$ Department of Physics, University of Central Florida, 12424 Research Parkway, \\ Suite 400, Orlando, FL 32826, USA \\ amasunov@mail.ucf.edu
}

\begin{abstract}
Mechanism of photoswitching in diarylethenes involves the lightinitiated symmetry-allowed disrotatory electrocyclic reaction. Here we propose a computationally inexpensive Density Functional Theory (DFT) based method that is able to produce accurate potential surfaces for the excited states. The method includes constrained optimization of the geometry for the ground and two excited singlet states along the ring-closing reaction coordinate using the Slater Transition State method, followed by single-point energy evaluation. The ground state energy is calculated with the broken-symmetry unrestricted Kohn-Sham formalism (UDFT). The first excited state energy is obtained by adding the UDFT ground state energy to the excitation energy of the pure singlet obtained in the linear response Time-Dependent (TD) DFT restricted Kohn-Sham formalism. The excitation energy of the double excited state is calculated using a recently proposed (Mikhailov, I. A.; Tafur, S.; Masunov, A. E. Phys. Rev. A 77, 012510, 2008) a posteriori Tamm-Dancoff approximation to the second order response TD-DFT.
\end{abstract}

Keywords: Time Dependent Density Functional Theory, Photochromism, Photoswitching, Optical Data Storage, Double excited state, Theoretical Photochemistry, Two-photon Absorption, Rational Materials design.

\section{Introduction}

Recording density of the data storage becomes an important issue in the recent years. While magnetic media neared its maximum capacity with the bit size c.a. $20 \mathrm{~nm}$, the technological advances in optical disks is expected to win the competition with traditional magnetic storage devices. In particular, these advances include the photon-mode recording, in the contrast to the optical memory systems presently available on the market. Most of the existing systems utilize heat-mode recording, where the light is converted into thermal energy, induces a magnetic or structural phase transition (magneto-optical [1,2] and phase-change [3] effects) and changes physical properties of the medium. In the photon-mode of data recording, the light initiates photochemical reaction of a particular component of the material. This allows introducing the third axial dimension to the recording process. This three-dimensional technology will use 
polymers, doped with the photochromic compounds undergoing a reversible photoisomerization [4]. A promising class of photochromics is exemplified by diarylethene compounds, shown in Scheme 1. They undergo photoinduced conrotatory ring opening and closing, and have important practical advantages over other classes of compounds, including thermal stability and resistance to linear optical photofatigue [5].

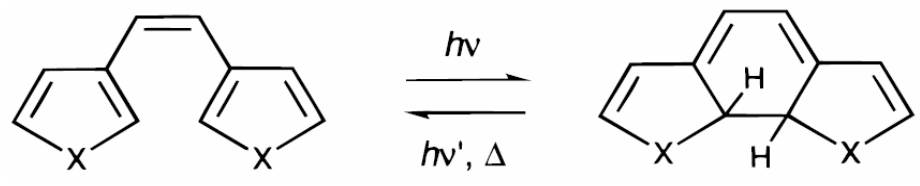

Scheme 1. Diarylethene photoswitching reaction $(\mathrm{X}=\mathrm{O}$ or $\mathrm{S})$

Some of the photochromic materials undergo photoisomerization in ultrafast regime (in the order of $10 \mathrm{fs}$ ). These ultrafast switching capabilities can be useful for various photonic devices, such as optical switches, variable frequency filters, attenuators, and phase shifters, interconnection, and components of optical computers.

Organic photonic materials have another important advantage, as their properties can be fine-tuned by chemical modifications of molecules. However, these modifications may change or completely eliminate the photochromic ability. Theoretical studies are indispensible to understand the reason of these changes and to formulate the guiding principles for the rational molecular design ([6] and [7]). However, as Nakamura et al. state in their recent review [7], the accurate relative energies of the excited states in real size molecules are still very difficult to calculate, because it requires the balanced description for both covalent (2A state) and ionic states (1B state).

In this contribution we propose a new theoretical method based on the Density Functional Theory. This method is able to produce accurate potential surfaces for the $1 \mathrm{~B}$ and $2 \mathrm{~A}$ excited states as compared to available experimental data and results of the high-level multireference wavefunction theory methods. It is also computationally inexpensive and capable to predict the photophysics of large molecules of practical interest.

\section{Theory}

Computational photochemistry offers a number of theoretical methods for investigation of photochemical reaction mechanisms. Unlike thermally activated chemical reactions, which take place in the ground electronic state $\left(\mathrm{S}_{0}\right)$, a photochemical process involves the electronically excited state $\left(\mathrm{S}_{1}\right)$. During this process the reactive system is electronically excited from $S_{0}$ to $S_{1}$, and after some evolution on an upper potential energy surface (PES) decays back to the ground state in either product or reactant basin through conical intersections (CIX). A useful simplified description of this process is called the reaction Pathway Approach [8]. Instead of the entire PES, it considers the minimum energy path (MEP) [9] which is followed by the center of a wave packet [10]. This approach is focused on local properties of PES, such as minima, barriers, and slopes. In the Pathway Approach, a Conical Intersection serves as a funnel, which 
delivers the excited state intermediate to the ground state reactant or the product, so that quantum yield is largely determined there [11]. It has been found very useful for a qualitative analysis of reaction mechanisms, prediction of photoproducts, and rationalization of experimental excited state lifetimes, quantum yields, absorption and emission spectra $[12,13]$.

A number of computational tools have been developed to predict the PES $[11,14,15]$. A computationally inexpensive approach to describe electronically excited systems is based on the time-dependent (TD) or, more precisely, linear response DFT formalism. Instead of orbital relaxation, TD-DFT uses a mathematically equivalent procedure where the KS wavefunction is expanded in terms of Slater determinants, singly excited with respect to the reference state. The rigorous formulation of TD-DFT [16] demonstrates that this description is in principle exact, given that the frequency-dependent exchange-correlation functional is known. In most practical applications, however, this frequency dependence is ignored (so called adiabatic TD-DFT). This method was often reported to accurately predict electronic spectra and excited state geometries. However, TD-DFT was found to be somewhat less successful in description of PESs in the vicinity of a CIX [17]. In this contribution we show that these difficulties are routed to the failure of the restricted Kohn-Sham formalism for the reference ground state close to geometry of the pericyclic minimum, and introduce a possible solution.

The Kohn-Sham formalism of DFT was developed for non-degenerate cases; it breaks down for systems with strong diradical character and degeneracy of the electronic levels, such as CIX geometries. However, static (also known as left-right) electron correlation can be taken into account by using different orbitals for different spin. This approach, known as the unrestricted Kohn-Sham formalism (UKS) is known to yield a qualitatively correct description of bond breaking [18].

Excited states, on the other hand, require the restricted formalism to avoid heavy mixing of higher spin states in description of the excited singlet. Although the TDDFT was suggested on the UKS reference [19], this is considered to be incorrect in a rigorous theory [20]. One possible approach for analyzing PES of excited states can be formulated by adding excitation energies obtained in the restricted TD-DFT formalism to the ground state energies calculated with the UKS method. Thereafter we will refer to this approach as to RTD-UDFT. Although for the photoswitching systems considered in this contribution the difference in the ground state energy obtained with the RKS and UKS formalisms is close to $20 \mathrm{kcal} / \mathrm{mol}$ or less, we will numerically show that this difference is sufficient to bring the excited state PESs to agreement with the results obtained at a higher theory level, when available.

Another theoretical development, necessary to describe the region of the conical intersection is related to the double excited states, missing in the adiabatic linear response TD-DFT approximation [21]. Mixing of the double excited states to the linear response TD-DFT states is offered by the Coupled Electronic Oscillator formalism [22-24], where doubly excited states appear in the second order as simple products of the excitations obtained at the linear response level. We recently used this fact to propose the a posteriori Tamm-Dancoff approximation (ATDA), and demonstrated its accuracy for linear polyenes in their ground state geometry [25]. We will show in Section 5, that ATDA-UDFT produces accurate energies for the double excited state in the entire range of the bond breaking reaction coordinate, provided that the molecular 
geometry corresponds to that state. For excited states that appear in the linear response TD-DFT the ATDA yields identical excitation energies and transition dipoles from the ground state, while permanent dipoles and state-to-state transition dipoles differ.

Since analytical gradients in the ATDA-UDFT approach are not yet implemented in computer codes, in our studies we use the Slater transition state method (STS) to optimize geometry of the excited states. In this method, half an electron is promoted from the highest occupied molecular orbital to the lowest unoccupied molecular orbital and self-consistency is achieved with these fractional orbital occupations [26]. STS is known to be a good approximation to the corresponding $\triangle \mathrm{SCF}$ excitation energy [27, 28]. Its further extension to the modified linear response DFT method [29] yields considerable improvement in description of the charge-transfer and Rydberg states, compared to the TD-DFT approach. A practical advantage of STS is an easier SCF convergence, compared to the excited-state SCF convergence, which often presents a major problem [30].

\section{Computational Details}

All calculations were performed using the Gaussian 2003 Rev. E1 suite of programs [31]. We used the hybrid meta-GGA exchange-correlation functional M05-2X from Truhlar's group with double fraction of the Hartree-Fock exchange, designed for accurate description of both equilibrium geometries and transition states. The minimum energy pathways (MEPs) were built using the relaxed scan along the forming pericyclic $\mathrm{C}-\mathrm{C}$ bond (reaction coordinate). The ground singlet state (S0), was optimized at the UM05-2X/6-31G level of theory, while the single $1 \mathrm{~B}$ and double $2 \mathrm{~A}$ excited singlet state geometries were optimized in the Slater Transition State method. STS was implemented using equal fractional occupation numbers for HOMO and LUMO in the alpha-set only [to approximate geometry of the single excited state $1 \mathrm{~B}$, $\operatorname{IOp}(5 / 75=1,76=2)]$, and both alpha and beta sets [to approximate geometry of the double excited state $2 \mathrm{~A}, \operatorname{IOp}(5 / 75=1,76=2,77=1,78=2)]$. Excitation energies were taken from single-point calculations in the a posteriori Tamm-Dancoff approximation for the lowest single excited state $1 \mathrm{~B}$ and the lowest double excited state $2 \mathrm{~A}$. The excitation energies thus obtained at the ATDA-M05-2X/6-31 level, were added to the ground state energies obtained at the UM05-2X/6-31G level of theory. The resulting ATDA-UM05-2X/6-31G//STS-UM05-2X/6-31G energies were plotted in the range of the reaction coordinate from 1.4 to $3.5 \mathrm{~A}$ with $0.1 \mathrm{~A}$ step size as MEPs.

\section{Results and Discussion}

Cyclohexadiene/hexatriene (CHD/HT) conversion is the simplest example of an electrocyclic reaction. Dynamics of cycloreversion in the CHD/HT system was repeatedly studied with time-resolved ultrafast spectroscopy techniques $[12,32]$. The results are summarized in Ref. [10].

The theoretical description of this process involves plotting realistic potential energy surfaces (PESs), which until recently required the use of ab initio multireferencebased quantum chemistry methods. Pioneering CAS study of the HT/CHD system 
was published by Robb, Olivucci et al. [33-35]. They found that 2A and 1A surfaces touch at a molecular conformation of tetraradical character, located away from the $C_{2^{-}}$ symmetric coordinate, and including partial bonds C1...C6 and C2...C6. They also found that accounting for dynamic electron correlation is essential to correctly predict the relative energies of $1 \mathrm{~B}$ and $2 \mathrm{~A}$ states. Despite the fact that $2 \mathrm{~A} / 1 \mathrm{~A}$ and $1 \mathrm{~B} / 2 \mathrm{~A}$ conical intersections complicate the energy landscape by adding an extra dimension, the qualitative interpretation given by the state correlation diagram along symmetric coordinate still holds. It was further confirmed at the high level (CASPT2 and MRCI) by building $a b$ initio PESs and performing two-dimensional quantum dynamics $[36,37]$ on these PESs. Barrierless descent on excited state PES was found to determine ultrafast photoconversion between CHD and HT, and quantum yield of this process was primarily determined by location of the 2A/1 A CIX.

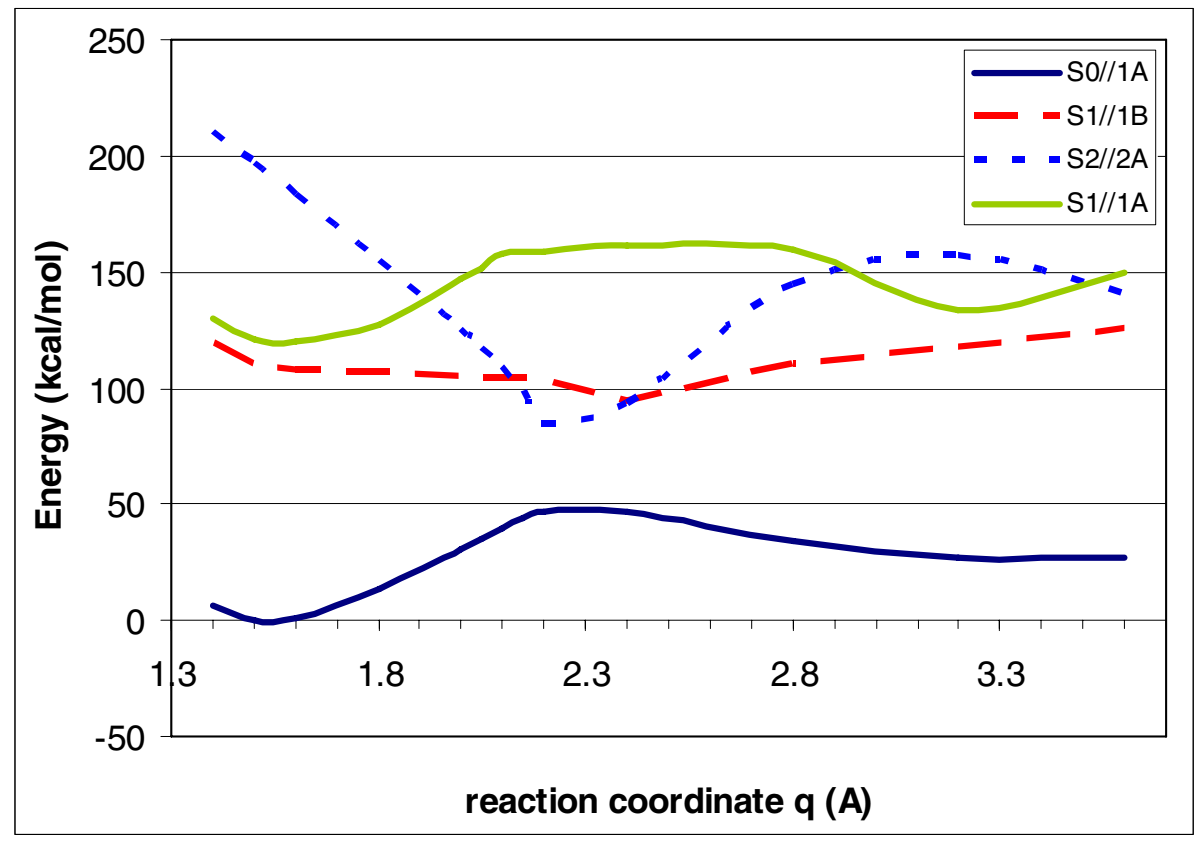

Fig. 1. Minimum energy pathways for the ground (S0, 1A), single excited (S1, 1B) and double excited states (S2, 2A) along the reaction pathway of the ring-closing $\mathrm{C}-\mathrm{C}$ bond in the $\mathrm{CHD} / \mathrm{HT}$ system, predicted at the ATDA-UDFT/6-31G//STS-DFT/6-31G theory level, using the M05-2X exchange-correlation functional. Absence of an appreciable energy barrier on the pathway from the 1B state of CHD in the Franck-Condon geometry (left) to the minimum on the 2A surface is consistent with ultrafast rate of the photoinitiated cycloreversion reaction $\mathrm{CHD} \rightarrow \mathrm{HT}$.

Minimum energy pathways (MEPs) obtained in this study are plotted in Fig. 1. As the $\mathrm{C} 1$...C6 reaction coordinate contracts from a non-bonding distance to the normal covalent bond, the bright $1 \mathrm{~B}$ state (characterized by the large transition dipole from the ground sate), is being monotonically stabilized in energy, starting descent to the pericyclic minimum. At the same time, the dark $2 \mathrm{~A}$ state (with a negligibly small 
transition dipole from the ground state) is being stabilized even faster, crosses the $1 \mathrm{~B}$ state surface, and forms the bottom of the pericyclic minimum.

It is worth noting that geometry of the ground state does not approximate the excited state geometry accurately enough to produce a reasonable potential energy surface. The vertical excitation curve, plotted in Fig. 1 and representing energy of the 1B state at the ground state geometry, displays a maximum instead of a pericyclic minimum, and contradicts both high level $a b$ initio and more accurate relaxed ATDAUDFT data.

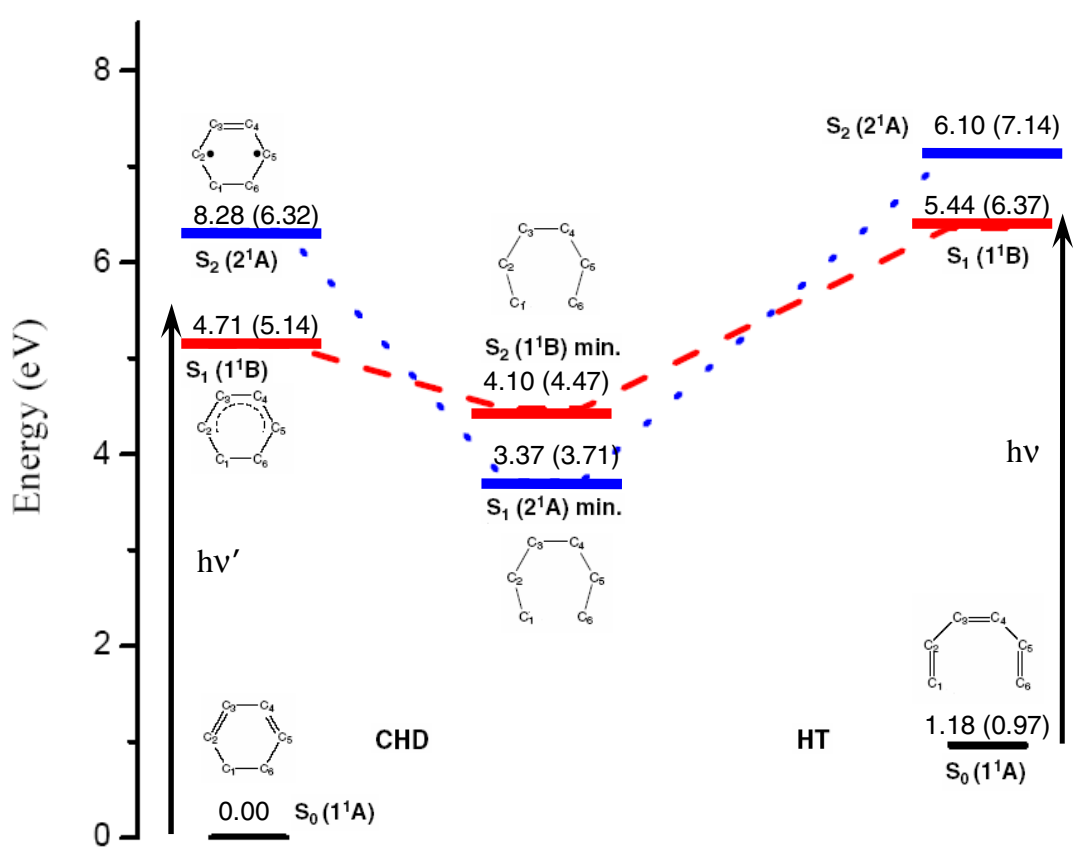

Fig. 2. Relative state energies (in $\mathrm{eV}$ ) for the ground and two lowest singlet excited states in CHD/HT system, obtained at the UDFT/6-31G//STS-DFT/6-31G theory level, using the M05$2 \mathrm{X}$ exchange-correlation functional. The results of high level MR-PT2 $a b$ initio calculations from Ref. [36] are shown for comparison in parentheses. Absence of an appreciable energy barrier on the pathway from the 1B state of CHD in the Franck-Condon geometry (left) to the minimum on the $2 \mathrm{~A}$ surface explains ultrafast rate of the photoinitiated cycloreversion reaction $\mathrm{CHD} \rightarrow \mathrm{HT}$.

A qualitative comparison between our modified DFT results and state-of-the-art wavefunction theory method MR-PT2 calculations of the CHD/HT system is presented in Fig. 2. Five important points were considered: ground state equilibrium geometries for closed and open isomers (CHD and HT), corresponding to Frank-Condon geometries of the ground states; and excited states $1 \mathrm{~B}$ and $2 \mathrm{~A}$, optimized into the respective pericyclic minima. They are in surprisingly good agreement with high level $a b$ initio results. As one can see, ATDA-UDFT //STS-DFT at the M05-2X/6-31G theory level, adopted in this work, almost uniformly overstabilizes 
both excited states by $0.4-1.0 \mathrm{eV}$, but retains the correct order of excited states, as compared to the multireference perturbation theory results. To the best of our knowledge, this is the first report of the correct state ordering in this system, obtained from a DFT-based approach.

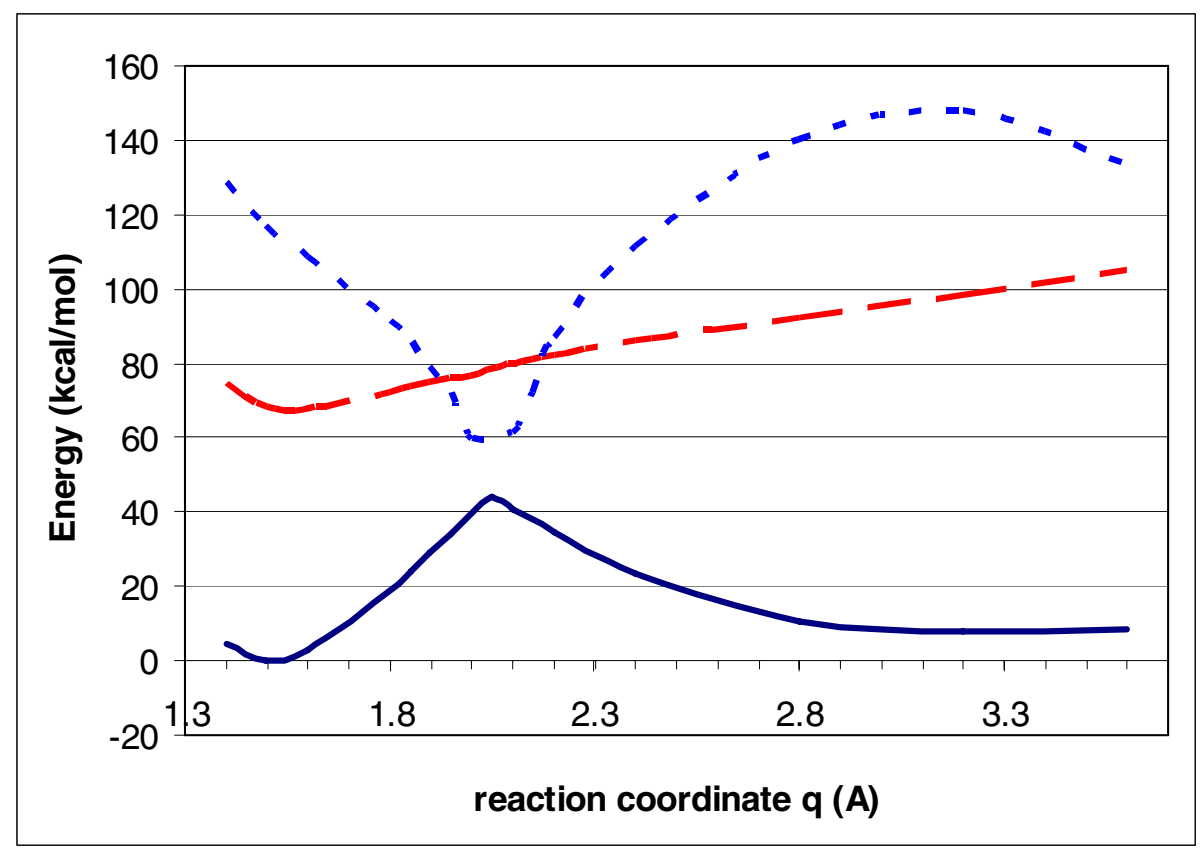

Fig. 3. Minimum energy pathways for the ground (S0, 1A), single excited (S1, 1B) and double excited states (S2, 2A) along the reaction pathway of ring-closing $\mathrm{C}-\mathrm{C}$ bond in the dithioarylethene system, predicted at the ATDA-UDFT/6-31G//STS-DFT/6-31G theory level, using the M05-2X exchange-correlation functional. The legend is the same as in Fig. 1. Absence of an appreciable energy barrier on the pathway from the $1 \mathrm{~B}$ state of the open form in the FranckCondon geometry (left) to the minimum on the $2 \mathrm{~A}$ surface is consistent with ultrafast rate of the photoinitiated cycloreversion reaction. The distance C1-C6 (reaction coordinate) was kept frozen during geometry optimizations of the ground and excited states using the STS-DFT method.

Dithienylethene (Scheme 1, with $\mathrm{X}=\mathrm{S}$ ) is the simplest homolog of diarilethens, an important class of compounds for photoswitching applications. Our calculations produced the minimum energy pathways for the ground and the two lowest single and double excited states, which are plotted in Fig. 3. One can see that MEP of the 1B state has a minimum in the closed form, and monotonically rises to the Franck-Condon region of the open form. The doubly excited 2A state, on the other hand, forms a pericyclic minimum and crosses below the $1 \mathrm{~B}$ state in the vicinity of the conical intersection.

Therefore, the excitation of the open form is followed by the ultrafast barrierless relaxation into pericyclic minimum along $1 \mathrm{~B}$ and then $2 \mathrm{~A}$ PES, while the excitation 
of the closed form will populate the potential minimum on the excited state surface. The conversion of the excited closed form into the pericyclic minimum must first overcome the small (c.a. $5 \mathrm{kcal} / \mathrm{mol}$ ) potential energy barrier, which leads to relatively slow cycloreversion. The excited state absorption will then bring the system from the $1 \mathrm{~B}$ to the $2 \mathrm{~A}$ state, followed by barrierless relaxation toward CIX. Thus, our MEPs explain both slow cycloreversion and ultrafast photoswitching upon sequential twophoton absorption.

\section{Conclusions}

A new approach to plot potential energy surfaces of the excited states, based on Density Functional Theory is presented. This approach includes both single and double excitations appearing in first and second order Time-Dependent DFT in the Coupled Electronic Oscillator formalism (dubbed the a posteriori Tamm-Dancoff approximation, ATDA-DFT). Unphysical spikes on these surfaces close to pericyclic minima were traced to the failure of the restricted Kohn-Sham formalism to describe the partial bond breaking on the ground states, and were eliminated by replacing the ground state energy component of the excited state with the one obtained in the unrestricted broken symmetry Kohn-Sham formalism (termed here ATDA-UDFT). Importance of excited state geometry optimization (as opposed to the habitual use of unrelaxed ground state geometries) in accurate prediction of these potential energy surfaces was demonstrated. For the lack of analytical derivatives at the ATDA-UDFT theory level the lowest single and double excited state geometry is approximated using the Slater Transition State method (STS-DFT). The combined ATDA-UDFT//STS-DFT approach was shown to slightly underestimate energy of both excited states but correctly reproduce the state ordering and the energy crossovers as compared to the high-level multireference perturbation theory results for hexatriene/cyclohexadiene system. The approach was also able to explain experimentally observed slow photochemical cycloreversion rates and fast excited state absorption initiated cycloreversion in model dithioarylethenes. This method may assist in future development of new photoswitching materials for advanced applications in the Information Technology.

Acknowledgements. This work is supported in part by the National Science Foundation (CCF 0740344). The authors are thankful to UCF Institute for Simulations and Training HPC Stokes facility, UCF I2Lab, and DOE NERSC for the generous donation of computer time. AEM acknowledges the ACS COMP Hewlett-Packard Outstanding Junior Faculty award presented for this work at the Fall 2008 American Chemical Society Meeting.

\section{References}

1. Kaneko, M.: Materials for magneto-optical recording. Mrs Bulletin 31, 314-317 (2006)

2. Wang, J.G., Sun, C.J., Hashimoto, Y., Kono, J., Khodaparast, G.A., Cywinski, L., Sham, L.J., Sanders, G.D., Stanton, C.J., Munekata, H.: Ultrafast magneto-optics in ferromagnetic III-V semiconductors. Journal of Physics-Condensed Matter 18, R501-R530 (2006) 
3. Zhou, G.F.: Materials aspects in phase change optical recording. Materials Science and Engineering a-Structural Materials Properties Microstructure and Processing 304, 73-80 (2001)

4. Kawata, S., Kawata, Y.: Three-dimensional optical data storage using photochromic materials. Chemical Reviews 100, 1777-1788 (2000)

5. Irie, M.: Diarylethenes for memories and switches. Chemical Reviews 100, 1685-1716 (2000)

6. Ern, J., Bens, A.T., Martin, H.D., Mukamel, S., Tretiak, S., Tsyganenko, K., Kuldova, K., Trommsdorff, H.P., Kryschi, C.: Reaction Dynamics of a Photochromic Fluorescing Dithienylethene. The Journal of Physical Chemistry A 105, 1741-1749 (2001)

7. Nakamura, S., Yokojima, S., Uchida, K., Tsujioka, T., Goldberg, A., Murakami, A., Shinoda, K., Mikami, M., Kobayashi, T., Kobatake, S., Matsuda, K., Irie, M.: Theoretical investigation on photochromic diarylethene: A short review. Journal of Photochemistry and Photobiology A: Chemistry 200, 10-18 (2008)

8. Garavelli, M.: Computational organic photochemistry: strategy, achievements and perspectives. Theoretical Chemistry Accounts 116, 87-105 (2006)

9. Truhlar, D.G., Gordon, M.S.: From Force-Fields to Dynamics - Classical and Quantal Paths. Science 249, 491-498 (1990)

10. Fuss, W., Hering, P., Kompa, K.L., Lochbrunner, S., Schikarski, T., Schmid, W.E., Trushin, S.A.: Ultrafast photochemical pericyclic reactions and isomerizations of small polyenes. Berichte Der Bunsen-Gesellschaft-Physical Chemistry Chemical Physics 101, 500-509 (1997)

11. Bernardi, F., Olivucci, M., Robb, M.A.: Potential energy surface crossings in organic photochemistry. Chemical Society Reviews 25, 321-328 (1996)

12. Fuss, W., Lochbrunner, S., Muller, A.M., Schikarski, T., Schmid, W.E., Trushin, S.A.: Pathway approach to ultrafast photochemistry: potential surfaces, conical intersections and isomerizations of small polyenes. Chemical Physics 232, 161-174 (1998)

13. Robb, M.A., Garavelli, M., Olivucci, M., Bernardi, F.: A computational strategy for organic photochemistry. Reviews in Computational Chemistry 15, 87-146 (2000)

14. Toniolo, A., Ben-Nun, M., Martinez, T.J.: Optimization of conical intersections with floating occupation semiempirical configuration interaction wave functions. Journal of Physical Chemistry A 106, 4679-4689 (2002)

15. Yarkony, D.R.: Marching along ridges. An extrapolatable approach to locating conical intersections. Faraday Discussions 127, 325-336 (2004)

16. Runge, E., Gross, E.K.U.: Density-Functional Theory for Time-Dependent Systems. Physical Review Letters 52, 997-1000 (1984)

17. Levine, B.G., Ko, C., Quenneville, J., Martinez, T.J.: Conical intersections and double excitations in time-dependent density functional theory. Molecular Physics 104, 1039-1051 (2006)

18. Gunnarsson, O., Lundqvist, B.I.: Exchange and Correlation in Atoms, Molecules, and Solids by Spin-Density Functional Formalism. Physical Review B 13, 4274-4298 (1976)

19. Cai, Z.L., Reimers, J.R.: Application of time-dependent density-functional theory to the (3)Sigma(-)(u) first excited state of H-2. Journal of Chemical Physics 112, 527-530 (2000)

20. Casida, M.E., Ipatov, A.: Excited-state spin-contamination in time-dependent densityfunctional theory for molecules with open-shell ground states. Abstr. Pap. Am. Chem. Soc. 231, 94-COMP (2006)

21. Neugebauer, J., Baerends, E.J., Nooijen, M.: Vibronic coupling and double excitations in linear response time-dependent density functional calculations: Dipole-allowed states of N-2. Journal of Chemical Physics 121, 6155-6166 (2004) 
22. Knoester, J., Mukamel, S.: Nonlinear optics using the multipolar hamiltonian - the blochmaxwell equations and local-fields. Physical Review A 39, 1899-1914 (1989)

23. Tretiak, S., Mukamel, S.: Density matrix analysis and simulation of electronic excitations in conjugated and aggregated molecules. Chemical Reviews 102, 3171-3212 (2002)

24. Tretiak, S., Chernyak, V.: Resonant nonlinear polarizabilities in the time-dependent density functional theory. Journal of Chemical Physics 119, 8809-8823 (2003)

25. Mikhailov, I.A., Tafur, S., Masunov, A.E.: Double excitations and state-to-state transition dipoles in pi-pi* excited singlet states of linear polyenes: Time-dependent densityfunctional theory versus multiconfigurational methods. Physical Review A 77, 012510 012511 (2008)

26. Slater, J.C.: Advances in Quantum Chemistry 6, 1-92 (1972)

27. Liberman, D.A.: Slater transition-state band-structure calculations. Physical Review B 62, 6851-6853 (2000)

28. Noodleman, L., Baerends, E.J.: Electronic-structure, magnetic-properties, electron-spinresonance, and optical-spectra for 2-fe ferredoxin models by lcao-X-alpha valence bond theory. Journal of the American Chemical Society 106, 2316-2327 (1984)

29. $\mathrm{Hu}, \mathrm{C}$., Sugino, O.: Average excitation energies from time-dependent density functional response theory. The Journal of Chemical Physics 126, 074112-074110 (2007)

30. Han, W.G., Liu, T.Q., Lovell, T., Noodleman, L.: Density functional theory study of $\mathrm{Fe}(\mathrm{IV}) \mathrm{d}-\mathrm{d}$ optical transitions in active-site models of class I ribonucleotide reductase intermediate $\mathrm{X}$ with vertical self-consistent reaction field method. Inorganic Chemistry 45, 8533-8542 (2006)

31. Frisch, M.J., et al.: Gaussian 2003. Gaussian, Inc., Wallingford (2004)

32. Kuthirummal, N., Rudakov, F.M., Evans, C.L., Weber, P.M.: Spectroscopy and femtosecond dynamics of the ring opening reaction of 1,3-cyclohexadiene. The Journal of Chemical Physics 125, 133307-133308 (2006)

33. Celani, P., Ottani, S., Olivucci, M., Bernardi, F., Robb, M.A.: What Happens During the Picosecond Lifetime of 2a(1) Cyclohexa-1,3-Diene - a Cas-Scf Study of the Cyclohexadiene Hexatriene Photochemical Interconversion. Journal of the American Chemical Society 116, 10141-10151 (1994)

34. Celani, P., Bernardi, F., Robb, M.A., Olivucci, M.: Do photochemical ring-openings occur in the spectroscopic state? B-1(2) pathways for the cyclohexadiene/hexatriene photochemical interconversion. Journal of Physical Chemistry 100, 19364-19366 (1996)

35. Garavelli, M., Celani, P., Fato, M., Bearpark, M.J., Smith, B.R., Olivucci, M., Robb, M.A.: Relaxation paths from a conical intersection: The mechanism of product formation in the cyclohexadiene/hexatriene photochemical interconversion. Journal of Physical Chemistry A 101, 2023-2032 (1997)

36. Tamura, H., Nanbu, S., Nakamura, H., Ishida, T.: A theoretical study of cyclohexadiene/hexatriene photochemical interconversion: multireference configuration interaction potential energy surfaces and transition probabilities for the radiationless decays. Chemical Physics Letters 401, 487-491 (2005)

37. Tamura, H., Nanbu, S., Ishida, T., Nakamura, H.: Ab initio nonadiabatic quantum dynamics of cyclohexadiene/hexatriene ultrafast photoisomerization. The Journal of Chemical Physics 124, 084313 (2006) 